\title{
Kinetic Theory for Some Intramolecular Reactions Proceeding on a Polymer Chain. II.*
}

\author{
Masahiko Sisıdo \\ Department of Polymer Chemistry, Kyoto University, Kyoto, Japan.
}

(Received September 7, 1972)

\begin{abstract}
Kinetic theory for the reactions proceeding on a polymer chain was developed in the field of polymer catalyst. Polymer catalyst is designed to possess either adsorption or catalytic site distributed along the chain. The former adsorbs a substrate and the complex formed subsequently undergoes an intramolecular reaction catalyzed by the latter to afford product and to regenerate free adsorption site. The overall reaction rate was formulated taking into account the distribution of catalytic sites in the neighborhood of each adsorption site. The reaction rate for polymer catalyst was compared with that for the corresponding low-molecular-weight catalyst. The acceleration by polymer catalyst was very marked when the reagent concentrations were low. A supplementary remark on Part I of this series was also made.
\end{abstract}

KEY WORDS Kinetic Theory / Polymer Catalyst / Intramolecular

Reaction / Matrix Method /

Catalysis in solution by various polymers containing both adsorption site $A$, and catalytic site $\mathrm{C}$, has been investigated by several workers. ${ }^{1-7}$ Most of these reactions were found to follow the Michaelis-Menten-type kinetics indicating adsorption of substrate $\mathrm{S}$, taking place prior to the product $(\mathrm{P})$-forming reaction. If the adsorption equilibrium (dissociation constant $K$ ) is established very rapidly, the rate of product-

$$
\mathrm{S}+\mathrm{A} \stackrel{K}{\rightleftarrows} \mathrm{AS} \stackrel{k_{\mathrm{I}}}{\longleftrightarrow} \mathrm{P}+\mathrm{A}
$$

forming reaction $\left(k_{\mathrm{I}}[\mathrm{AS}]\right)$ may be described by the sum of the intramolecular unimolecular reaction rates of adsorbed substrates AS, catalyzed by catalytic groups attached to the same polymer chain.

Although some complicated situations have been noted in practice, e.g., intramolecular aggregation in polymer catalyst, ${ }^{1-3}$ bifunctional interactions of pendent catalytic groups, ${ }^{4-7}$ it seems useful to formulate a kinetic theory for rather simplified cases. In this paper it is assumed that the adsorption equilibrium is attained rapidly and the adsorption occurs by the short-range binding forces excluding coulombic

* Hereafter the previous paper appeared in Polymer J., 3, 84 (1972) will be read as the Part I of the series. force. Then the rate of reaction catalyzed by polymer catalyst can be formulated using a method analogous to that described in Part I of this series, where intrachain reactions between substrates and catalytic species, both being chemically bonded to the polymer chain, were treated.

\section{FORMULATION OF KINETIC THEORY}

Case 1. Kinetics Considering the Probability of Finding a Distribution of Catalytic Group is Derived from the Independent Probabilities for Each Residue

To begin with, the simplest case that was treated in Part I will be described here briefly. In this case it is assumed that the probability $p_{I}$ for the $I$-th residue ( $I$-th monomer unit counting from a chain end) to carry a catalytic group is independent of the states of other residues. Then, the overall rate of product formation is written as

$$
\text { rate }=\mathrm{d}[\mathrm{P}] / \mathrm{d} t=\sum_{I, J=1}^{N} k_{I J} p_{J}[\mathrm{AS}]_{I}
$$

where $N$ is the total number of residues in a chain and $[\mathrm{AS}]_{I}$ is the concentration of substrates adsorbed on the $I$-th residue. $k_{I J}$ is the first-order rate constant for a reaction between 
$\mathrm{AS}_{I}$ and a catalytic group attached to the $J$-th residue. For sufficiently long chains, $k_{I J}$ depends only upon the separation $(i=|I-J|)$ between $I$-th and $J$-th residues. Hence, for the uniform distribution of catalytic groups $\left(p_{J}=p\right)$, eq 1 is written as

$$
\text { rate }=2 p \sum_{i=1}^{N-1}(N-i) k_{i}[\mathrm{AS}]_{I}
$$

Furthermore, for the uniform distribution of adsorbed substrate

$$
\text { rate }=2 p \sum_{i=1}^{Z} k_{i}[\mathrm{AS}]
$$

[AS] is the total concentration of adsorbed substrate and $Z$ denotes the reaction range, that is, the effective separation between $I$-th and $J$-th residues where the reaction actually takes place. In eq $3 N \gg Z$ is also assumed.

Case 2. Kinetics for the Case where the Distribution of Catalytic Group is Described by a Markov Process

In this section the case will be treated in which the probability to find a catalytic group on the $i$-th residue is dependent on the state of $(i-1)$-th residue. Thus the conditional probability is defined as $p\left(n_{i-1}, n_{i}\right)$ for all $i$, where $n_{i}$ is the number (zero or unity) of catalytic group attached on the $i$-th residue. Here a residue carrying a substrate is numbered as the zero-th residue. As in Part I, the probability $W(\boldsymbol{n})$ for a reaction range to have the distribution of catalytic group, $n=\left(n_{-Z}, \ldots, n_{Z}\right)$ is ${ }^{8}$

$$
\begin{aligned}
W(\boldsymbol{n})= & p\left(n_{0}, n_{-1}\right) \cdots p\left(n_{-Z+1}, n_{-Z}\right) \\
& \times p\left(n_{0}, n_{1}\right) \cdots p\left(n_{Z-1}, n_{Z}\right)
\end{aligned}
$$

Apparent first-order rate constant for a substrate in the reaction range $\boldsymbol{n}$ is ${ }^{8}$

$$
K(\boldsymbol{n})=\sum_{i=-Z}^{Z} k_{i}\left(n_{i-1}, n_{i+1}\right) n_{i}
$$

where $k_{i}\left(n_{i-1}, n_{i+1}\right)$ is the first-order rate constant for the reaction of a substrate catalyzed by a catalytic group attached to the $i$-th residue. The rate constant depends on the neighboring residues $\left(n_{i-1}\right.$ and $\left.n_{i+1}\right)$. The total rate is written as

$$
\text { rate }=\sum_{n} W(\boldsymbol{n}) K(\boldsymbol{n})[\mathrm{AS}]
$$

Introducing eq 4 and 5 into eq 6, and using the same method as adopted in Part $I$, the following equation was obtained, in which $n_{i}$ is replaced by $m_{i}=n_{i}+1$.

$$
\begin{aligned}
\text { rate } /[\mathrm{AS}]= & 2\left[\sum_{m_{-Z}}\left\{\mathbf{P}^{Z}\right\}_{m_{0}, m_{-}}\right] \\
& \times\left[\sum_{i=1}^{Z} \sum_{m_{Z}}\left\{\mathbf{P}^{i-1} \mathbf{Q}_{i} \mathbf{P}^{Z-i}\right\}_{m_{0}, m_{Z}}\right]
\end{aligned}
$$

where matrices $\mathbf{P}$ and $\mathbf{Q}_{i}$ are

$$
\begin{gathered}
\mathbf{P}=\left[\begin{array}{cc}
p(0,0) & p(0,1) \\
p(1,0) & p(1,1)
\end{array}\right] \\
\mathbf{Q}_{i}=\left[\begin{array}{cc}
p(0,1) k_{i}(0,0) p(1,0) & p(0,1) k_{i}(0,1) p(1,1) \\
p(1,1) k_{i}(1,0) p(1,0) & p(1,1) k_{i}(1,1) p(1,1)
\end{array}\right]
\end{gathered}
$$

$\mathbf{P}$ is a transition matrix and the normalization conditions are

$$
p(0,0)+p(0,1)=1, \quad p(1,0)+p(1,1)=1
$$

Using these conditions eq 7 is transformed into eq 11 .

$$
\text { rate }=2 \sum_{i=1}^{Z} \sum_{m_{Z}}\left\{\mathbf{P}^{i-1} \mathbf{Q}_{i}\right\}_{m_{0}, m_{Z}}[\mathrm{AS}]
$$

Alternatively, introducing super matrices ${ }^{9}$

$$
\text { rate }=2[\mathrm{AS}] \sum_{m_{Z}}\left\{\left[\begin{array}{ll}
\mathbf{E}_{2} & \mathbf{0}
\end{array}\right] \prod_{i=1}^{Z}\left[\begin{array}{ll}
\mathbf{P} & \mathbf{Q}_{i} \\
\mathbf{0} & \mathbf{E}_{2}
\end{array}\right]\left[\begin{array}{c}
\mathbf{0} \\
\mathbf{E}_{2}
\end{array}\right]\right\}_{m_{0}, m_{Z}}
$$

where $\mathbf{E}_{2}$ and $\mathbf{0}$ are the unit matrix and zero matrix of the second order, respectively. If $k_{i}$ is independent of $n_{i-1}$ and $n_{i+1}$, eq 11 becomes

$$
\text { rate }=2[\mathrm{AS}] \sum_{i=1}^{Z}\left\{\mathbf{P}^{i}\right\}_{m_{0}, 2} k_{i}
$$

Substituting $p\left(n_{i-1}, n_{i}\right)$ for $p$ for all $i$, eq 13 is the same as eq 3 as expected. The above equations represent the microscopic meanings of the rate constant $k_{\mathrm{I}}$ for the intramolecular reaction of adsorbed substrate.

Although the above treatment was developed for the infinite long chains, this restriction can be easily eliminated if the uniform distribution of adsorbed substrate is assumed. The term $\sum_{m_{Z}}\left\{\mathbf{P}^{i-1} \mathbf{Q}_{i}\right\}_{m_{0}, m_{Z}}$ in eq 11 represents the contribution of the $i$-th residue to the overall rate, and the number of the pairs of residues separated from one another by $i$ residues is $(N-i)$. Therefore 


\section{Sisido}

$$
\text { rate }=2 \sum_{i=1}^{N-1} \frac{(N-i)}{N} \sum_{m_{Z}}\left\{\mathbf{P}^{i-1} \mathbf{Q}_{i}\right\}_{m_{0}, m_{Z}}[\mathrm{AS}]
$$

And if $k_{i}$ is independent of $n_{i-1}$ and $n_{i+1}$

$$
\text { rate }=2 \sum_{i=1}^{N-1} \frac{(N-i)}{N}\left\{\mathbf{P}^{i}\right\}_{m_{0}, 2} k_{i}[\mathrm{AS}]
$$

Using eq 14 or 15 , the dependence of reaction rate upon the molecular weight of polymer catalyst can be discussed.

Case 3. Kinetics for Substrates Chemically

\section{Bonded to a Polymer Chain}

Intramolecular reactions of substrates chemically bonded to a polymer chain, not in equilibrium with free substrates, have been treated in the Part I in detail. However the reaction rate has not been derived yet. This quantity can be obtained by the differentiation of the fraction of unreacted substrate $y$, by time $t$. $y$ is written as $^{8}$

$$
y=\left[\sum_{m_{Z}}\left\{\mathbf{A}_{1} \cdots \mathbf{A}_{Z}\right\}_{m_{0}, m_{Z}}\right]^{2}
$$

where matrices $\mathbf{A}_{i}$ 's are

$$
\mathbf{A}_{i}=\left[\begin{array}{ll}
p(0,0) & p(0,1) \exp \left(-k_{i} t\right) \\
p(1,0) & p(1,1) \exp \left(-k_{i} t\right)
\end{array}\right]
$$

Differentiation of eq 16 with respect to time yields

$$
\begin{aligned}
\mathrm{d} y / \mathrm{d} t= & 2\left[\sum_{m_{Z}}\left\{\mathbf{A}_{1} \cdots \mathbf{A}_{Z}\right\}_{m_{0}, m_{Z}}\right] \\
& \times\left[\sum_{m_{Z}} \sum_{i}\left\{\mathbf{A}_{1} \cdots\left(\mathbf{d} \mathbf{A}_{i} / \mathbf{d} t\right) \cdots \mathbf{A}_{Z}\right\}_{m_{0}, m_{Z}}\right]
\end{aligned}
$$

The above equation becomes simpler using matrices $\mathbf{B}_{i}$ 's.

$$
\mathbf{B}_{i}=-\mathrm{d} \mathbf{A}_{i} / \mathrm{d} t=\left[\begin{array}{ll}
0 & k_{i} p(0,1) \exp \left(-k_{i} t\right) \\
0 & k_{i} p(1,1) \exp \left(-k_{i} t\right)
\end{array}\right]
$$

Using super matrices, ${ }^{9}$ eq 20 is obtained.

$$
\begin{aligned}
-\mathrm{d} y / \mathrm{d} t & =2\left[\sum_{m_{Z}}\left\{\mathbf{A}_{1} \cdots \mathbf{A}_{Z}\right\}_{m_{0}, m_{Z}}\right] \\
& \times\left[\sum_{m_{Z}}\left\{\left[\begin{array}{ll}
\mathbf{E}_{2} & \mathbf{0}
\end{array}\right] \prod_{i=1}^{Z}\left[\begin{array}{ll}
\mathbf{A}_{i} & \mathbf{B}_{i} \\
\mathbf{0} & \mathbf{A}_{i}
\end{array}\right]\left[\begin{array}{c}
\mathbf{0} \\
\mathbf{E}_{2}
\end{array}\right]\right\}_{m_{0}, m_{Z}}\right]
\end{aligned}
$$

The initial rate can be obtained by substituting zero for $t$ in eq 20 .

$$
(-\mathrm{d} y / \mathrm{d} t)_{t=0}=2 \sum_{i=1}^{Z}\left\{\mathbf{P}^{i}\right\}_{m_{0}, 2} k_{i}
$$

Equation 21 can be utilized to determine the absolute values of $k_{i}$ 's from the initial rate of the intramolecular reaction. For short chains eq 21 becomes

$$
(-\mathrm{d} y / \mathrm{d} t)_{t=0}=2 \sum_{i=1}^{N-1} \frac{(N-i)}{N}\left\{\mathbf{P}^{i}\right\}_{m_{0}, 2} k_{i}
$$

\section{DISCUSSION}

The Efficiency of Polymer Catalyst Compared with the Corresponding Low-Molecular-Weight Catalyst

In most of the polymer catalysts the efficiency is not so high as enzyme. ${ }^{1-7,10}$ The ratio of reaction rates catalyzed by a polymer catalyst to that catalyzed by a corresponding lowmolecular-weight catalyst can be estimated if the change of substrate reactivity by the adsorption is ignored. In this respect the first case will be discussed for the simplest situation. Putting $K$ as the dissociation constant of adsorbed substrate, the total concentration of adsorbed substrate is

$$
\begin{aligned}
{[\mathrm{AS}]=} & (1 / 2)\left[\left([\mathrm{S}]_{\mathrm{t}}+a\left[\mathrm{C}_{\mathrm{p}}\right] / p+K\right)\right. \\
& \left.-\left\{\left([\mathrm{S}]_{t}+a\left[\mathrm{C}_{\mathrm{p}}\right] / p+K\right)^{2}-4 a[\mathrm{~S}]_{\mathrm{t}}\left[\mathrm{C}_{\mathrm{p}}\right] / p\right\}^{1 / 2}\right]
\end{aligned}
$$

where $[S]_{\mathrm{t}}$ is the total concentration of substrates including adsorbed ones and $\left[\mathrm{C}_{\mathrm{p}}\right]$ is the total concentration of catalytic groups attached to the chain. The mole fraction of adsorption sites in the chain is denoted by $a$. Combining the above equation with eq 3 , the rate of reaction using a polymer catalyst is obtained. On the other hand, a usual second-order rate of the reaction between free substrates and free catalytic species is

$$
k_{\mathrm{II}}[\mathrm{S}]_{\mathrm{t}}[\mathrm{C}]
$$

where the second-order rate constant is denoted by $k_{\mathrm{II}}$. If the reaction rates are compared at $\left[\mathrm{C}_{\mathrm{p}}\right]=[\mathrm{C}]$, the ratio of reaction rates $E_{\mathrm{f}}$, is

$$
\begin{aligned}
E_{\mathrm{f}}= & (1 / 2)\left[\left(p /[\mathrm{C}]+a /[\mathrm{S}]_{\mathrm{t}}+K p /[\mathrm{C}][\mathrm{S}]_{\mathrm{t}}\right)\right. \\
& -\left\{\left(p /[\mathrm{C}]+a /[\mathrm{S}]_{\mathrm{t}}+K p /[\mathrm{C}][\mathrm{S}]_{\mathrm{t}}\right)^{2}\right. \\
& \left.\left.-4 a p /[\mathrm{C}][\mathrm{S}]_{\mathrm{t}}\right\}^{1 / 2}\right]\left[2 \sum_{i=1}^{Z} k_{i} / k_{\mathrm{II}}\right]
\end{aligned}
$$

According to Goodman and Morawetz, ${ }^{11-13}$ 


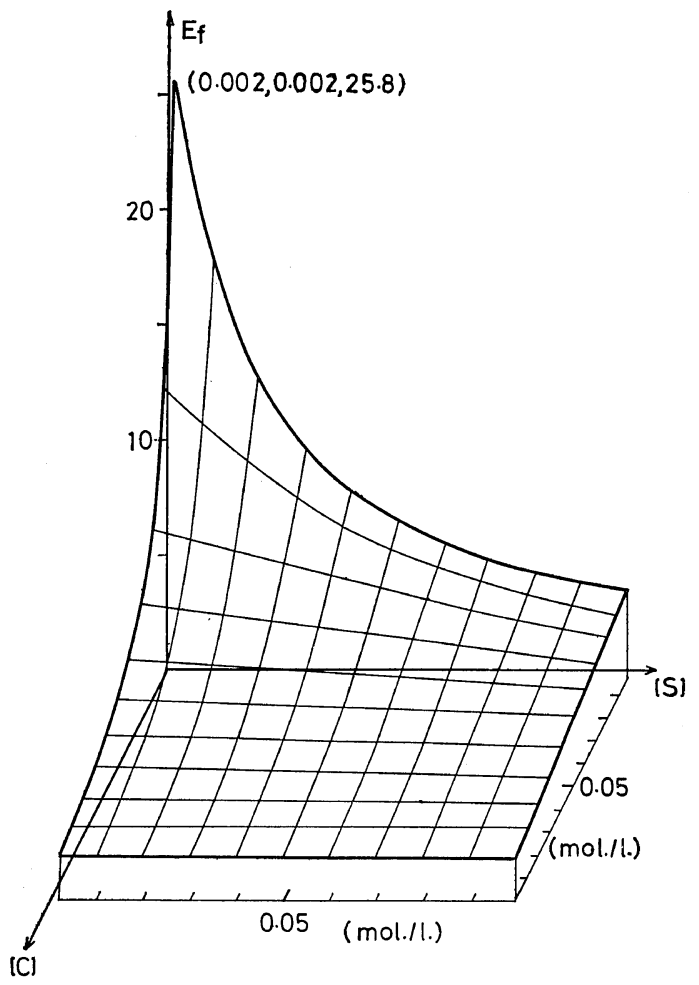

Figure 1. Three-dimensional diagram for the efficiency of a polymer catalyst. $E_{\mathrm{f}}=$ ratio of reaction rates catalysed by polymer catalyst against that by the corresponding low-molecular-weight catalyst: $K, 0.01(\mathrm{~mol} / l), p, 0.1 ; a, 0.2$. [S $]_{\mathrm{t}}$ represents total concentration of substrate, and $[C]$ represents total concentration of catalytic group.

the maximum effective concentration $\left(C_{\mathrm{eff}}^{\max }=\right.$ $\left.2 \sum_{i} k_{i} / k_{\mathrm{II}}\right)$ takes a value from 2 to 5 for polyacrylamide and polymethacrylamide chains. The theoretical value of $C_{\mathrm{eff}}^{\max }$ calculated by them agreed in order with the experimental value. ${ }^{11-12}$ However the experimental results by Stoll and Rouve $^{14-15}$ and the calculation using a $g-t$-rotational isomer model by the author ${ }^{16}$ showed that the value of $k_{i} / k_{\text {II }}$ for short polymethylene chain were about $1 / 10$ as low as the value expected by the method of Goodman and Morawetz. Therefore, the exact value of $C_{\mathrm{eff}}^{\max }$ is still obscure. Here it is tentatively assumed to be 2 and the numerical calculation of eq 25 was performed in the following conditions

$$
K=0.01(\mathrm{~mol} / l) \quad p=0.1 \text { and } a=0.2
$$

Results are shown in Figure 1. The acceleration of the reaction by polymer catalyst is very marked when the reagent concentrations are low.

\section{Physical Significance of Dissociation Constant}

If the rate of conformational transitions of polymer segment is much faster than the dissociation rate of adsorbed substrates, the polymer segments in the neighborhood of an adsorption site can move carrying the substrate on it. In this case the dissociation constant measured in the equilibrium state can be applied to eq 25 . On the other hand, if the dissociation rate is faster than the rate of conformational transition, the concentration of adsorbed substrates sufficiently close to a catalytic group may differ from the concentration expected from the dissociation constant determined under the equilibrium condition. In this case the dissociation constant in eq 25 must be an effective value, which is usually obtained by a kinetic treatment, such as the Lineweaver-Burk plot. ${ }^{17}$

Acknowledgment. The author is indebted to Professor T. Higashimura and Dr. Y. Imanishi for helpful discussions.

\section{REFERENCES}

1. T. Kunitake, F. Shimada, and C. Aso, J. Amer. Chem. Soc., 91, 2716 (1969).

2. T. Kunitake and S. Shinkai, ibid., 93, 4247 (1971).

3. T. Kunitake and S. Shinkai, ibid., 93, 4256 (1971).

4. C. G. Overberger, M. Morimoto, I. Cho, and J. C. Salamone, Macromolecules, 2, 553 (1969).

5. C. G. Overberger and M. Morimoto, J. Amer. Chem. Soc., 93, 3222 (1971).

6. C. G. Overberger, M. Morimoto, I. Cho, and J. C. Salamone, ibid., 93, 3228 (1971).

7. C. G. Overberger and J. C. Salamone, Account. Chem. Res., 2, 217 (1969).

8. M. Sisido, Polymer J., 3, 84 (1972).

9. P. J. Flory, "Statistical Mechanics of Chain Molecules," Interscience Publishers, Inc., New York, N.Y., 1969, Chapter 3.

10. W.P. Jencks, "Catalysis in Chemistry and Enzymology,' McGraw-Hill, Inc., 1969, Chapter 1.

11. N. Goodman and H. Morawetz, J. Polym. Sci., Part C, 31, 177 (1970).

12. N. Goodman and H. Morawetz, ibid., Part A-2, 
M. Sisido

9, 1657 (1971)

13. H. Morawetz, Account. Chem. Res., 3, 354 (1970).

14. M. Stoll and A. Rouvé, Helv. Chim. Acta, 18, 1087 (1935).
15. H. Morawetz and N. Goodman, Macromolecules, 3, 699 (1970).

16. M. Sisido, ibid., 4, 737 (1971).

17. H. Lineweaver and D. Burk, J. Amer. Chem. Soc., 56, 658 (1934). 